International Journal of Pure and Applied Mathematics

Volume 106 No. 2 2016, 631-637

ISSN: 1311-8080 (printed version); ISSN: 1314-3395 (on-line version)

url: http://www.ijpam.eu

doi: 10.12732 /ijpam.v106i2.25

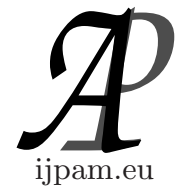

\title{
NUMERICAL TREATMENT OF THE RLC SMOOTHING CIRCUIT USING LEAPFROG METHOD
}

\author{
S. Sekar ${ }^{1}$, M. Vijayarakavan ${ }^{2}$ \\ ${ }^{1}$ Department of Mathematics \\ Government Arts College (Autonomous) \\ Salem, 636 007, Tamil Nadu, INDIA \\ ${ }^{2}$ Department of Mathematics \\ V.M.K.V. Engineering College \\ Salem, 636 308,Tamil Nadu, INDIA
}

\begin{abstract}
In this paper, We develop the Leapfrog method for solving RLC smoothing circuit [4]. The obtain discrete solutions were compared with another method taken from the literature [4]. The results obtained using Leapfrog is found to be very closer to the original solutions of these problems. Error graphs for the obtained results and exact solutions are presented in a graphical form to highlight the efficiency of this method. This Leapfrog method can be easily implemented in a digital computer and the solution can be obtained for any length of time.
\end{abstract}

AMS Subject Classification: 65L80, 65L05

Key Words: state space equation, differential equations, initial value problems, single term Haar wavelet series, Leapfrog method

\section{Introduction}

Mathematical modelling aims to describe the different aspects of the real world, their interaction, and their dynamics through mathematics. It constitutes the

Received: October 23, 2015

Published: February 18, 2016

$\S_{\text {Correspondence author }}$ (c) 2016 Academic Publications, Ltd.

url: www.acadpubl.eu 
third pillar of science and engineering, achieving the fulfilment of the two more traditional disciplines, which are theoretical analysis and experimentation. Now days, mathematical modelling has a key role also in fields such as the environment and industry, while its potential contribution in many other areas is becoming more and more evident. One of the reasons for this growing success is definitely due to the impetuous progress of scientific computation; this discipline allows the translation of a mathematical model, which can be explicitly solved only occasionally, into algorithms that can be treated and solved by ever more powerful computers.

Single-term Haar wavelet series methods have become very popular, both as computational techniques as well as subject for research, which were discussed by Sekar et al. [1, 2, 4, 5, 6]. Palanisamy et al.[3] analysed the smoothing circuits using single-term Walsh series (STWS). Recently Sekar and his team of researchers analyzed second order linear system with singular-A, Second-order linear singular systems, first order linear fuzzy differential equations and first order linear singular systems using Leapfrog method [1, 2, 7, 8]. The objective of this article is to use the Leapfrog method to solve RLC smoothing circuit (discussed by Sekar et al. [4],). The paper is organized as follows: in Section 2 we describe the Leapfrog method required for our subsequent development. In Section 3 general formation of the RLC smoothing circuit with example presented and we apply the proposed numerical method to the hybrid fuzzy differential equations and report our numerical finding and demonstrate the accuracy of the proposed method.

\section{Leapfrog Method}

The most familiar and elementary method for approximating solutions of an initial value problem is Eulers Method. Eulers Method approximates the derivative in the form of $y^{\prime}=f(t, y), y\left(t_{0}\right)=y_{0}, y \in R^{d}$ by a finite difference quotient $y^{\prime} \approx(y(t+h)-y(t)) / h$. We shall usually discretize the independent variable in equal increments:

$$
t_{n+1}=t_{n}+h, \quad n=0,1, \ldots, t_{0} .
$$

Henceforth we focus on the scalar case, $N=1$. Rearranging the difference quotient gives us the corresponding approximate values of the dependent variable:

$$
y_{n+1}=y_{n}+h f\left(t_{n}, y_{n}\right), \quad n=0,1, \ldots, t_{0}
$$


To obtain the leapfrog method, we discretize $t_{n}$ as in $t_{n+1}=t_{n}+h, n=$ $0,1, \ldots, t_{0}$, but we double the time interval, $h$, and write the midpoint approximation

$$
y(t+h)-y(t) \approx h y^{\prime}\left(t+\frac{h}{2}\right)
$$

in the form

$$
y^{\prime}(t+h) \approx(y(t+2 h)-y(t)) / h
$$

and then discretize it as follows:

$$
y_{n+1}=y_{n-1}+2 h f\left(t_{n}, y_{n}\right), \quad n=0,1, \ldots, t_{0}
$$

The leapfrog method is a linear $m=2$-step method, with $a_{0}=0, a_{1}=$ $1, b_{-1}=-1, b_{0}=2$ and $b_{1}=0$. It uses slopes evaluated at odd values of $n$ to advance the values at points at even values of $n$, and vice versa, reminiscent of the childrens game of the same name. For the same reason, there are multiple solutions of the leapfrog method with the same initial value $y=y_{0}$. This situation suggests a potential instability present in multistep methods, which must be addressed when we analyze themtwo values, $y_{0}$ and $y_{1}$, are required to initialize solutions of $y_{n+1}=y_{n-1}+2 h f\left(t_{n}, y_{n}\right), n=0,1, \ldots, t_{0}$ uniquely, but the analytical problem $y^{\prime}=f(t, y), y\left(t_{0}\right)=y_{0}, y \in R^{d}$ only provides one. Also for this reason, one-step methods are used to initialize multistep methods.

\section{The RLC Smoothing Circuit}

In this section, The output of a digital-to-analogue converter is a staircase function in a digital communication system as shown in Figure 1.

To recover the true analogue signal the output must be smoothed. The RLC circuit shown in Figure 2 is used for this purpose (Subbayyan et al [9]). For the above RLC smoothing circuit, the corresponding differential equations are:

$$
\left.\begin{array}{l}
\frac{d i}{d t}=-i(t)-v(t)+e(t), \\
\frac{d v}{d t}=i(t),
\end{array}\right\}
$$

with initial condition $i(0)=0 ; v(0)=0$. where $i(t)$ is the circuit current, $v(t)$ is the output voltage and the input $e(t)$ is given in Figure 1. 


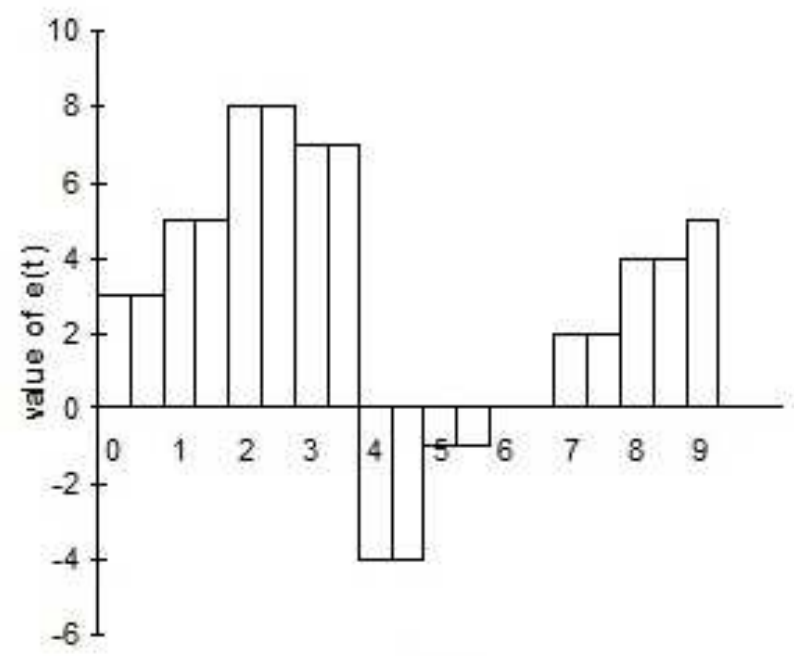

time

Figure 1. Input voltage (Staircase fundion)

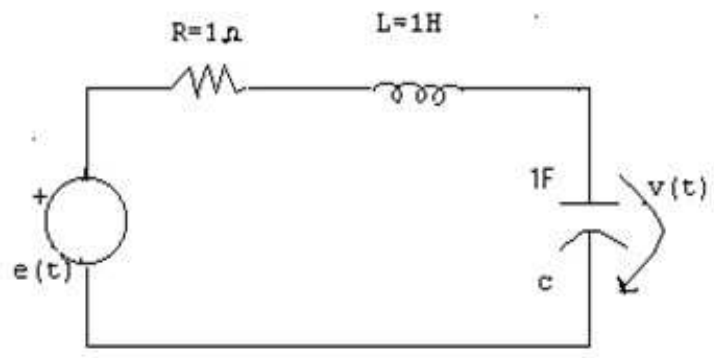

Figure 2. An RLC smoothing circuit

\section{Numerical Examples}

In this section, we solved the RLC smoothing circuit problem to show the efficiency and accuracy of the proposed methods. The above equation can be represented in matrix form as :

$$
\left[\begin{array}{l}
i^{\prime}(t) \\
v^{\prime}(t)
\end{array}\right]=\left[\begin{array}{cc}
-1 & -1 \\
1 & 0
\end{array}\right]\left[\begin{array}{l}
i(t) \\
v(t)
\end{array}\right]+\left[\begin{array}{l}
1 \\
0
\end{array}\right] e(t),
$$

which is of the form $x^{\prime}(t)=A x(t)+B u(t)$ 
The exact solution for (1) is

$$
\begin{gathered}
i(t)=\frac{2}{\sqrt{3}} e(t) \exp \left(-\frac{t}{2}\right) \sin \left(-\frac{\sqrt{3}}{2} t\right), \\
v(t)=e(t)\left[1-\exp \left(-\frac{t}{2}\right)\left(\cos \left(\frac{\sqrt{3}}{2} t\right)+\sin \left(\frac{\sqrt{3}}{2} t\right)\right)\right] .
\end{gathered}
$$

The exact solution for $\mathrm{i}(\mathrm{t})$ and $\mathrm{v}(\mathrm{t})$ and the corresponding discrete solution curves obtained by Palanisamy and Arunachalam using STWS [3] with fixed initial condition. It is observed that, the error between the exact solution and the discrete solution determined by the above authors is more due to the fixed initial conditions $\mathrm{i}(0)=0$ and $\mathrm{v}(0)=0$ throughout the interval of the staircase function.

\begin{tabular}{cccccc}
\hline & \multicolumn{2}{c}{ STHWS Error } & & \multicolumn{2}{c}{ Leapfrog Error } \\
\cline { 2 - 3 } \cline { 5 - 6 }$t$ & $i(t)$ & $v(t)$ & & $i(t)$ & $v(t)$ \\
\hline 0 & 0 & 0 & & 0 & 0 \\
1 & $1 \mathrm{E}-05$ & $1 \mathrm{E}-05$ & & $1.01 \mathrm{E}-07$ & $1.01 \mathrm{E}-07$ \\
2 & $2 \mathrm{E}-05$ & $2 \mathrm{E}-05$ & & $2.01 \mathrm{E}-07$ & $2.01 \mathrm{E}-07$ \\
3 & $3 \mathrm{E}-05$ & $3 \mathrm{E}-05$ & & $3.01 \mathrm{E}-07$ & $3.01 \mathrm{E}-07$ \\
4 & $4 \mathrm{E}-05$ & $4 \mathrm{E}-05$ & & $4.01 \mathrm{E}-07$ & $4.01 \mathrm{E}-07$ \\
5 & $5 \mathrm{E}-05$ & $5 \mathrm{E}-05$ & & $5.01 \mathrm{E}-07$ & $5.01 \mathrm{E}-07$ \\
6 & $6 \mathrm{E}-05$ & $6 \mathrm{E}-05$ & & $6.01 \mathrm{E}-07$ & $6.01 \mathrm{E}-07$ \\
7 & $7 \mathrm{E}-05$ & $7 \mathrm{E}-05$ & & $7.01 \mathrm{E}-07$ & $7.01 \mathrm{E}-07$ \\
8 & $8 \mathrm{E}-05$ & $8 \mathrm{E}-05$ & & $8.01 \mathrm{E}-07$ & $8.01 \mathrm{E}-07$ \\
9 & $9 \mathrm{E}-05$ & $9 \mathrm{E}-05$ & & $9.01 \mathrm{E}-07$ & $9.01 \mathrm{E}-07$ \\
10 & $1 \mathrm{E}-04$ & $1 \mathrm{E}-04$ & & $1.01 \mathrm{E}-06$ & $1.01 \mathrm{E}-06$ \\
\hline
\end{tabular}

Table 1: Error calculations

As the input $\mathrm{e}(\mathrm{t})$ is a staircase function, one has to initialize the initial condition for every sub-interval in the numerical methods, only then it will agree well with the exact solution. Also in this paper two more methods STHWS and Leapfrog method have been applied to the RLC smoothing circuit problem (1). And the corresponding discrete solutions have been determined with interval size $\mathrm{h}=0.01$. The absolute errors between the exact solutions and discrete solutions obtained by the methods STHWS and Leapfrog method have been determined and represented in the Table 1. 


\section{Conclusions}

It is observed from the above example of smoothing circuit that the solution obtained by STHWS and Leapfrog method coincide very well with the exact solution with an error tolerance 10-7. To find the numerical solution of a system of linear differential equations involving staircase function, one has to change the initial starting value for every sub-interval (i.e. wherever the function is discontinuous). This will be achieved only when the exact solutions are known, otherwise the numerical solution obtained for solving the system of linear differential equations involving staircase function leads to more error, refer Table 1.

Moreover, it is observed that the execution time, number of computations involved and the number of bytes required are very less in Leapfrog method when compared to STHWS. Above all, the accuracy is more in Leapfrog method than the other method.

\section{Acknowledgments}

The authors gratefully acknowledge the Dr. A. Murugesan, Assistant Professor, Department of Mathematics, Government Arts College (Autonomous), Cherry Road, Salem - 636 007, for his encouragement and support. The authors also thank Dr. S. Mehar Banu, Assistant Professor, Department of Mathematics, Government Arts College for Women, Salem - 636 008, Tamil Nadu, India, for her kind help and suggestions.

\section{References}

[1] S. Karunanithi, S. Chakravarthy and S. Sekar. Comparison of Leapfrog and single term Haar wavelet series method to solve the second order linear system with singular-A. Journal of Mathematical and Computational Sciences, 4:804-816, 2014.

[2] S. Karunanithi, S. Chakravarthy and S. Sekar. A Study on Second-Order Linear Singular Systems using Leapfrog Method. International Journal of Scientific and Engineering Research, 5:747-750, 2014.

[3] K. R. Palanisamy and V. P. Arunachalam. Analysis of smoothing circuits using a singleterm Walsh series. International Journal of Electronics, 59(2):225-230, 1985.

[4] S. Sekar and G.Balaji. A Study on RLC Smoothing Circuit using Single-term Haar wavelet series. International Journal of Physical Sciences, 22(2M): 381-386, 2010.

[5] S. Sekar and A. Manonmani. A Study On Time-Varying Singular Nonlinear Systems Via Single-Term Haar Wavelet Series. International Review of Pure and Applied Mathematics, 5(2):435-441, 2009. 
[6] S. Sekar and A. Manonmani. A Study On Second Order Mechanical Vibratory Systems Using Single-Term Haar Wavelet Series. International Journal of Logic Based Intelligent Systems, 3(2):197-202, 2009.

[7] S. Sekar and K. Prabhavathi. Numerical solution of first order linear fuzzy differential equations using Leapfrog method. IOSR Journal of Mathematics, 10:07-12, 2014.

[8] S. Sekar and M. Vijayarakavan. Numerical Investigation of first order linear Singular Systems using Leapfrog Method. International Journal of Mathematics Trends and Technology, 12:89-93, 2014.

[9] R. Subbayyan and K. M. Zakariah. Computer aided design of electronic circuits using single term Walsh series. International Journal of Electronics, 54: 161-165, 1983. 
